\title{
Nudges: diseño y evaluación
}

\section{Nudges: design and evaluation}

\author{
Elena Costas Pérez \\ KSNET y ESADE (España) \\ ORCID: https://orcid.org/0000-0002-5992-3008 \\ elena.costas@ksnet.eu
}

\section{NOTA BIOGRÁFICA}

Doctora en Economía por la Universidad de Barcelona, máster por la University College of London. Ha trabajado como investigadora en la UAB y el Banco Inter-Americano de Desarrollo, y como analista de políticas en la Organización para la Cooperación y el Desarrollo Económicos (OCDE). Es socia fundadora de Knowledge Sharing Network (KSNET) y profesora de ESADE. Sus áreas de investigación son la economía política y la gobernanza.

\section{NOTA BIOGRÁFICA}

Máster en Políticas Públicas y Sociales por la Universidad Pompeu Fabra y Máster en Economía Aplicada por la Universidad Torcuato Di Tella de Argentina. Actualmente trabaja como investigador en Knowledge Sharing Network (KSNET) en la evaluación de políticas públicas. Anteriormente, ha trabajado como consultor en temas de vivienda social y asesor económico en organismos públicos de la Argentina.

\section{RESUMEN}

La literatura sobre nudges y su evaluación es extensa, y destaca la diversidad de sesgos de comportamiento y posibles intervenciones. Los nudges buscan modificar la arquitectura de las decisiones individuales, intentando cambiar el comportamiento de las personas en una dirección concreta y a un bajo coste. Su impacto debe tener en cuenta el coste-efectividad del dinero invertido, en comparación a otras intervenciones públicas más tradicionales que persiguen el mismo objetivo. El uso de experimentos en su evaluación ha convertido a los nudges en una importante herramienta de la política basada en la evidencia, mejorando así el diseño y efectividad de la acción pública. En este artículo buscamos revisar el conocimiento actual en torno al diseño y evaluación de los nudges, y su importancia para el desarrollo de políticas basadas en evidencia.

\section{PALABRAS CLAVE}

Economía del comportamiento; políticas públicas; nudges; ensayos controlados aleatorizados; política basada en evidencia.

\section{ABSTRACT}

The literature on nudges and their evaluation is extensive, and it highlights the diversity of behavioural biases and possible interventions. Nudges seek to modify the architecture of individual decisions. They 
GAPP. Nueva Época - N. ${ }^{\circ}$ 25, marzo 2021 - ISSN: 1989-8991 - DOI: https://doi.org/10.24965/gapp.i25.10868 - [Págs. 8-22] Número monográfico - Los nudges y el diseño conductual de políticas públicas

change people's behavior in a specific direction and at a low cost. Its impact must take into account the cost-effectiveness of the money invested, compared to other traditional public interventions that pursue the same objective. The use of experiments in its evaluation has made nudges an important tool of evidence-based policy, thus improving the design and effectiveness of public action. In this article we seek to review the current knowledge regarding the design and evaluation of nudges, and its importance for the development of evidence-based policies.

\section{KEYWORDS}

Behavioural economics; public policy; nudges; randomized controlled trial; evidence-based policy.

\section{SUMARIO}

1. INTRODUCCIÓN. 2. NUDGES Y POLÍTICAS PÚBLICAS. 2.1. ECONOMÍA DEL COMPORTAMIENTO

Y POLÍTICAS BASADAS EN EVIDENCIA. 2.2. SESGOS Y NUDGES. 3. ¿CÓMO DISEÑAR NUDGES?

I. DEFINICIÓN DEL PROBLEMA. II. DIAGNÓSTICO. III. DISEÑO. REFERENCIAS BIBLIOGRÁFICAS.

\section{INTRODUCCIÓN ${ }^{1}$}

¿Por qué algunas personas no se vacunan, fuman o beben en exceso aun sabiendo que es negativo para su salud?, ¿A qué se debe que algunos adolescentes abandonen el sistema educativo prematuramente?, ¿Cómo evitar que las personas tiren basura en las playas o parques públicos? Las debilidades, y grandezas, del comportamiento humano son esenciales para comprender problemáticas en dominios tan diversos como la salud, la educación, o el medio ambiente, pero también la criminalidad, la movilidad, la pobreza y tantos otros. Entender la manera en que los seres humanos toman decisiones es imprescindible para diseñar intervenciones que tengan como finalidad mejorar la vida de los mismos.

El enfoque tradicional al momento de diseñar políticas públicas ha sido considerar a los seres humanos como agentes perfectamente racionales que cuentan con toda la información disponible (Bhargava y Loewenstein, 2015). Un individuo de estas características comprende perfectamente los incentivos, tanto positivos como negativos, así como las consecuencias de sus actos ${ }^{2}$. Ante este tipo de agentes, el mecanismo más efectivo para lograr el comportamiento deseado radica en centrarse exclusivamente en los incentivos y sanciones, o en los costos y beneficios que generan una determinada conducta (García Arteagoitia, 2019).

Sin embargo, las ciencias del comportamiento, un campo de investigación que reconoce la existencia de otros factores, más allá de la racionalidad, en la toma de decisiones de los agentes, han demostrado que, muy frecuentemente, las personas no se comportan del modo descripto anteriormente (Simon, 1956). Por esta razón, a lo largo de las últimas décadas, las políticas públicas han estado fuertemente vinculadas al estudio del comportamiento humano, entendiendo así las limitaciones cognitivas en el proceso de toma de decisiones de los distintos agentes (Lindblom, 1959; Straßheim y Beck, 2019).

En el año 2008 se publicó el libro fundacional de esta corriente de pensamiento: Nudge: Improving Decisions About Health, Wealth, and Happiness (Thaler y Sunstein, 2009). El libro, que describe intervenciones públicas basadas en nudges, se convirtió en un bestseller. Los nudges (pequeños «empujones» o «codazos») son intervenciones que buscan modificar la arquitectura de las decisiones individuales, intentando cambiar el comportamiento de las personas en una dirección concreta; pero, y esto es importante, sin prohibir ninguna opción, ni alterar en gran medida los incentivos económicos. Se trata de acciones baratas de implementar, que no son órdenes coercitivas para los ciudadanos, sino que inducen a un comportamiento previsible. (Thaler y Sunstein, 2009). Su objetivo es poner los hallazgos de las ciencias del comportamiento al servicio del sector público. Por ejemplo, un nudge podría basarse en hacer que los alimentos saludables sobresalgan en el comedor de un colegio o de una empresa, con el objetivo de que los individuos mejoren su dieta.

1 Los autores agradecen al equipo editorial de la revista la publicación de este artículo, así como los comentarios y sugerencias de los evaluadores anónimos que han mejorado su estructura y contenido.

2 Vale señalar que este paradigma no considera que los individuos no se equivocan, sino que no lo hacen sistemáticamente. 
El éxito de los nudges no se limitó a la venta de libros. Dos años después, el Reino Unido creó el Behavioural Insight Team (BIT), o «Unidad de Nudges», ahora independiente del gobierno. También el gobierno estadounidense creó el Social and Behavioral Science Team (SBST), seguido en 2015 por la Office of Evaluation Sciences (OES). Hoy en día son muchos los países que cuentan con equipos dedicados a usar las ciencias del comportamiento para mejorar la administración y las políticas públicas.

Los nudges en las políticas públicas implican usar el conocimiento derivado de las ciencias económicas, la neurociencia y la psicología, para incentivar o desincentivar actuaciones concretas de los distintos agentes, y alcanzar así objetivos establecidos por los dirigentes públicos. Al modificar el entorno en el que los ciudadanos toman decisiones, teniendo en cuenta los mecanismos de comportamiento que se activan en cada caso, los nudges constituyen un importante instrumento de políticas públicas en la acción de gobierno. Sin embargo, los nudges, así como las distintas herramientas de comportamiento aplicadas a las políticas públicas, pueden generar dudas respecto a sus dimensiones éticas, su eficiencia o sus limitaciones. Por esta razón, el objetivo de este artículo es revisar cuál es el conocimiento actual sobre el diseño de los nudges y qué podemos aprender de su evaluación. Para ello, describimos en primer lugar la relación entre los nudges y las políticas públicas. Después analizamos las distintas fases en el proceso de diseño e implementación de los nudges. En el cuarto apartado examinamos cómo podemos evaluar la efectividad de los nudges. Finalmente, presentamos una serie de reflexiones sobre el rol actual de los nudges y su importancia para el diseño de políticas públicas.

\section{NUDGES Y POLÍTICAS PÚBLICAS}

\subsection{Economía del comportamiento y políticas basadas en evidencia}

Desde la publicación de Thaler y Sunstein (2009) las intervenciones públicas vinculadas a los nudges y la ciencia del comportamiento se han convertido en una herramienta habitual en el toolkit de muchos gobiernos. Como hemos visto, se han creado diferentes unidades gubernamentales para potenciar su diseño e implementación. En este sentido, uno de los factores que han ayudado a potenciar la popularidad de los nudges es su relación con las políticas basadas en la evidencia (Behavioural Insights Team, 2010; Einfeld, 2019; Straßheim et al., 2015).

La implementación de políticas basadas en evidencia parte del supuesto de que la evidencia sólida constituye una condición necesaria para la elaboración de políticas públicas. La aplicación de evidencia rigurosa permitiría diseñar políticas y programas más efectivos y eficientes (Davies et al., 2000). Por lo tanto, si la eficacia de las políticas públicas depende en gran medida del comportamiento humano, sus decisiones y acciones (Barrows et al., 2018), un diseño basado en la evidencia debe fundamentarse en una adecuada comprensión de la conducta humana, es decir, deben tenerse en cuenta los sesgos y limitaciones conductuales. En este modelo de pensamiento, los nudges suponen un instrumento de política pública poderoso.

El uso de experimentos o ensayos controlados aleatorizados (RCT por sus siglas en inglés) para evaluar la eficiencia de los nudges, que se analizan con más detalle en la sección 4.1, ha contribuido en gran medida a que constituyan un importante elemento de políticas públicas basadas en la evidencia. Se trata de una forma sencilla y barata de analizar el potencial impacto que una aplicación a mayor escala de esa acción podría tener, lo que consigue ahorrar dinero público y tener evidencias claras de su efectividad. Por lo tanto, la experimentación localizada (Sabel y Zeitlin, 2012) de los nudges como elemento de la economía del comportamiento es una de las principales razones por las que este instrumento ha ganado popularidad entre los gobiernos como una medida de política basada en la evidencia.

Al incorporar la evidencia sobre el comportamiento de los individuos, y sus limitaciones cognitivas, en el diseño de las políticas públicas, debemos tener en cuenta que Simon (1956) ya señalaba que los humanos poseen racionalidad limitada y que, si bien tienen la capacidad de hallar soluciones satisfactorias, no presentan la capacidad de optimizar, en el sentido que asume el homoeconomicus. Posteriormente, muchos de estos aspectos fueron sistematizados y popularizados por Kahneman (2011), quien explica el comportamiento humano a través de dos sistemas de pensamiento. Por un lado, el sistema 1, o automático, que actúa involuntariamente. El sistema 1 permite, por ejemplo, percibir las distancias, el origen de un sonido repentino, hacer cálculos extremadamente simples o detectar el estado de ánimo a partir de una voz. Por otro lado, tenemos el sistema 2, o reflexivo, que opera de manera lenta, 
controlada, y que requiere esfuerzo a nuestro cerebro. Este sistema permite, por ejemplo, desarrollar cálculos complejos, centrar la atención en lo que dice nuestro interlocutor o comprobar la validez de un argumento lógico.

Ambos sistemas interactúan para definir el comportamiento y la toma de decisiones de las personas. Así, el sistema 1 genera intuiciones que son respaldadas o corregidas por el sistema 2. Sin embargo, en ocasiones erramos en nuestras decisiones: utilizamos el sistema 1, que genera sesgos, cuando deberíamos utilizar el sistema 2 .

Este modelo de pensamiento, que tiene en cuentas los sesgos y las limitaciones conductuales, es más preciso para explicar el comportamiento humano que el paradigma de perfecta racionalidad (Shafir, 2007). Por lo tanto, un enfoque de políticas basadas en evidencia debería incorporar las ciencias del comportamiento en el diseño de políticas públicas. Asimismo, la aplicación de las ciencias del comportamiento al diseño de políticas públicas puede dar lugar a intervenciones con elevado coste-efectividad, ya que, generalmente, el costo de aplicar intervenciones conductuales es sumamente bajo y simple en relación con el potencial impacto que puede generar (Bhargava y Loewenstein, 2015).

\subsection{Sesgos y nudges}

Hasta el momento se ha hablado de sesgos, pero ¿a qué nos referimos concretamente? Los sesgos son desviaciones sistemáticas del pensamiento racional. Es decir, se trata de errores que ocurren de manera regular para la mayoría de los seres humanos. Por supuesto, algunos factores, como la experiencia o el conocimiento, pueden ayudar a mitigar los efectos de estos sesgos. Sin embargo, la naturaleza humana hace que todas las personas estemos expuestos en mayor o menor medida a ellos.

En gran medida, la literatura científica del campo de las ciencias del comportamiento se ha centrado en identificarlos, analizar cómo se producen, en qué condiciones estos sesgos son más frecuentes, y si es posible generar mecanismos para evitarlos o, al menos, moderar sus efectos (Samson, 2020; Della Vigna y Linos, 2020; Dhami, 2016; Hummel y Maedche, 2019).

Se ha identificado una gran variedad de sesgos y existen diversas clasificaciones de los mismos (Barrows et al., 2018; De Gilder et al., 2005; IRS, 2017; OECD, 2019). Comprender cada uno y aprender a identificarlos permite señalar aquellas instancias donde las políticas públicas pueden fallar por no adaptarse al comportamiento humano. Por esta razón, a continuación, se mencionan algunos de los más habituales, clasificados de acuerdo a si afectan las preferencias, las creencias o la forma en que procesamos de la información ${ }^{3}$ :

\section{Sesgos de preferencias}

- Aversión a las pérdidas: sufrimos más por perder una cantidad de dinero que el beneficio que experimentamos por ganar esa misma cantidad. Esto puede ser de utilidad, por ejemplo, para diseñar una política para reducir el uso de bolsas desechables. Así, un impuesto por utilizar las bolsas tendría un efecto superior que una bonificación de la misma cuantía ofrecida a cambio de su utilización (Homonoff et al., 2018).

- Cortoplacismo: preferimos las gratificaciones inmediatas porque valoramos más los eventos presentes que los futuros. Elegimos un beneficio menor hoy frente a un beneficio mayor mañana. Esto explica la dificultad para dejar de fumar (Giné et al., 2010) o para aumentar los ahorros destinados a la jubilación (Hershfield et al., 2011).

- Inconsistencia temporal: sufrimos el «sesgo del presente». Por ejemplo, una persona preferirá recibir $10 €$ hoy a recibir $11 €$ en una semana. Sin embargo, si ambos beneficios se demoran un año, es decir, recibir $10 €$ en un año o recibir $11 €$ en un año y una semana, la persona seguramente seleccionará esta última opción (O'Donoghue y Rabin, 1999). Esto se explica porque las personas tienen dificultades para establecer con precisión sus preferencias futuras (Ariely y Loewenstein, 2006) y aprender de experiencias pasadas (Kahneman, 1994).

3 Esta clasificación ha sido propuesta por BRAIN, el departamento del Banco Interamericano de Desarrollo dedicado a la implementación de las ciencias del comportamiento en el diseño de políticas públicas (https://www.iadb.org/en/research-and-data/behavioralresources). 
- Inercia o sesgo del status-quo: queremos conservar el estado actual de las cosas, a menos que existan importantes incentivos para cambiar. Esto ocurre aun cuando los costos de modificar la situación actual son sumamente reducidos (Samuelson y Zeckhauser, 1988). Este sesgo explica, por ejemplo, que mantengamos suscripciones a planes que ya no nos resultan de utilidad.

- Normas sociales: seguimos las reglas implícitas (valores, acciones y expectativas) que señalan el comportamiento esperado dentro de una sociedad.

- Reciprocidad: respondemos a las acciones de los demás con una acción equivalente, sea esta positiva o negativa (Fehr y Gächter, 2000).

\section{Creencias}

- Exceso de confianza: sobreestimamos nuestra propia capacidad para realizar una determinada actividad (Pallier et al., 2002). La falacia de la planificación, por la cual subestimamos el tiempo que nos demandará una tarea, es un claro ejemplo (Buehler et al., 1994).

- Exceso de optimismo: subestimamos la probabilidad de eventos negativos, como contagiarnos de determinadas enfermedades, y sobreestimamos la probabilidad de eventos positivos, como ganar la lotería (Sharot, 2011).

- Heurística ${ }^{4}$ de disponibilidad: asignamos una mayor probabilidad a eventos futuros basándonos en disponibilidad de información de los mismos que tenemos ahora (Tversky y Kahneman, 1974).

- Heurística de representatividad: extrapolamos las características de los objetos o eventos conocidos a los desconocidos (Kahneman y Tversky, 1972). Por ejemplo, tendemos a juzgar la calidad de un producto por el precio: dado que los productos de calidad suelen tener un precio elevado, asumimos que los productos caros suelen ser de buena calidad.

\section{Procesamiento de la información}

- Efecto encuadre (framing): sacamos conclusiones diferentes dependiendo de cómo se nos presenta la información. Por ejemplo, una misma información puede presentarse con un enfoque de pérdidas o de ganancias -una apuesta con $90 \%$ de probabilidades de perder vs. una apuesta con $10 \%$ de probabilidades de ganar-. Si bien el resultado es el mismo, los diferentes enfoques producen diferentes reacciones (Kahneman y Tversky, 1979).

- Prominencia: nos atrae lo que destaca. Aplica tanto a lo que sobresale, como a lo que deja de sobresalir (IRS, 2017).

- Preocupaciones (hassle factors): pequeños factores o inconvenientes impiden que tomemos decisiones buenas para nosotros. Por ejemplo, cómo se presenta la información para solicitar una ayuda pública, o el hecho de que debamos acudir a acciones o instancias adicionales, hace que no la pidamos (Bettinger et al., 2012).

- Sobrecarga cognitiva: nuestra capacidad de atención y memoria es limitada, con lo que no podemos procesar toda la información disponible (Mullainathan y Shafir, 2013). Por ejemplo, una sobrecarga de opciones nos fatiga, y tomamos decisiones erróneas o las diferimos indefinidamente (lyengar y Lepper, 2000).

- Efecto halo: atribuimos cualidades o destrezas a una persona a partir de la percepción global que tengamos de ella, aún cuando esas cualidades no tengan ninguna relación con esa persona (Nisbett y Wilson, 1977).

Estos sesgos permiten visualizar cómo el contexto afecta la correcta toma de decisiones. Precisamente, este contexto constituye lo que Thaler y Sunstein (2009) denominan la «arquitectura de decisiones», una arquitectura que puede ser moldeada de modo tal de mejorar o facilitar la toma de decisiones por parte de las personas a través de nudges. Esto es particularmente relevante cuando las personas necesitan tomar decisiones que son complejas e infrecuentes, o cuyo feedback no se hace sentir de forma inmediata (Thaler y Sunstein, 2009). Tales situaciones incluyen, por ejemplo, decidir cuánto dinero ahorrar para la jubilación (Benartzi y Thaler, 2007), la elección de un seguro de salud (Abaluck y Gruber, 2011), o el comportamiento frente al consumo eléctrico y la contaminación ambiental (Schultz et al., 2007).

\footnotetext{
4 Los heurísticos son atajos mentales o reglas rápidas que nos ayudan a tomar decisiones.
} 


\section{3. ¿CÓMO DISEÑAR NUDGES?}

La arquitectura de esas decisiones es maleable. Sin embargo, alterarlas con nudges implica riesgos éticos y para la libertad de las personas (Battaglio et al., 2019). El diseño de nudges debe realizarse, por tanto, teniendo en cuenta dos principios básicos. Primero, el «paternalismo libertario»: el arquitecto del nudge debe procurar influir el comportamiento sólo para mejorar la calidad de vida de la gente, a juicio de ellas mismas. Y las personas deben ser libres de tomar las decisiones que deseen, aun si resultan prejudiciales para ellas mismas. Por esa razón, los nudges deben ser fácilmente evitables. El segundo principio es el de compatibilidad entre estímulo y respuesta. La idea es que el estímulo o nudge que se aplique sea coherente con la acción o respuesta deseada.

Como hemos visto, el potencial de los nudges ha provocado el surgimiento de numerosas organizaciones y áreas dentro de las Administraciones Públicas dedicadas a formular e introducir elementos conductuales en las políticas públicas (Afif et al., 2018). Estas «unidades de comportamiento» han desarrollado diversas metodologías para diseñar intervenciones conductuales. Si bien existen algunas diferencias, la mayoría de ellas contemplan una serie de fases básicas similares a las que se describen a continuación.

\section{i. Definición del problema}

Esta fase busca determinar la problemática que pretende abordarse del modo más preciso posible: quiénes son las personas afectadas por esta problemática, qué elementos caracterizan a estos colectivos, cuantificarlos y, si corresponde, delimitar territorialmente dónde se encuentran localizados.

El objetivo de esta primer fase es conocer la situación actual y establecer cuál es el comportamiento deseado (Martínez Villarreal et al., 2020). Un problema mal definido conducirá a un mal diseño de política. Si bien esto es válido para cualquier tipo de política, en este caso puede motivar respuestas conductuales no deseadas, lo que puede dar lugar a una amplificación de la problemática.

En este sentido, la investigación preliminar de estadísticas o datos administrativos puede ser de utilidad para confirmar y dimensionar posibles problemáticas. Este análisis permitirá generar hipótesis sobre comportamientos específicos de grupos de personas que pueden estar resultando problemáticos, ya sea por el comportamiento en sí mismo -una mala alimentación-, o porque afecta al desarrollo de una política -reduce el acceso a un programa público-. Esta hipótesis no debe abordar las posibles causas, ya que esto corresponde a la posterior fase de diagnóstico, sino que debe centrarse estrictamente en identificar qué comportamientos subyacen a la problemática (Barrows et al., 2018).

\section{ii. Diagnóstico}

La fase de diagnóstico tiene como finalidad determinar las causas que generan los comportamientos asociados a la problemática. En este sentido, comprender el contexto resulta primordial para reconocer posibles barreras -como la falta de dinero o infraestructura, o desviaciones sistemáticas del comportamiento- que generan la problemática.

Para llevar a cabo este diagnóstico, es recomendable empezar realizando una revisión sistemática de la literatura sobre el tema, la cual, además de brindar información sobre la problemática, puede sugerir posibles intervenciones, un diseño de evaluación y métodos de investigación adecuados (IRS, 2017).

Después, es aconsejable llevar a cabo un análisis de las políticas que se hayan implementado, así como también de los datos cualitativos y cuantitativos existentes sobre la problemática (Van Bavel et al., 2013). En algunas ocasiones, este análisis puede confirmar que se han aplicado las medidas tradicionales correctamente, por lo cual debería revisarse si existen elementos conductuales que explican la problemática. Para ello, existen diversas técnicas que permiten analizar detalladamente el comportamiento de las personas $^{5}$. Generalmente, se basan en la elaboración de un esquema que describe todos los puntos de decisión y acción que ocurren antes, durante y posteriormente al comportamiento analizado. Este esquema se suele presentar como un mapa de procesos y permiten identificar las áreas donde potencialmente se pueden introducir intervenciones conductuales (OECD, 2019).

5 Algunas de estas técnicas comprenden el «user journey mapping», una técnica habitual entre los diseñadores de experiencia del usuario (UX), o el «behavioural mapping», utilizado por Ideas42. 
Luego, para cada uno de esos puntos de acción o decisión, se analizan e identifican los factores psicológicos y características contextuales que podrían afectar la conducta (Barrows et al., 2018). Por ejemplo, se analiza el entorno físico, como señales visuales que pueden desviar la atención, o aspectos actitudinales, que pueden reflejar situaciones de stress.

Es posible que se detecten diversas barreras conductuales potencialmente problemáticas, por lo que será necesario escoger una, o varias de ellas, para abordarlas desde la economía del comportamiento (Martínez Villarreal et al., 2020). Una forma de seleccionar las barreras sobre las cuales intervenir es recurrir a la propia revisión de la literatura para determinar cuáles son las más relevantes. No obstante, en algunos casos, pueden existir otro tipo de consideraciones como la factibilidad, el potencial impacto, o incluso factores de carácter ético, político o financiero que determinen qué barreras priorizar (OECD, 2019).

\section{iii. Diseño}

Una vez seleccionada la barrera conductual que se desea abordar, es recomendable investigar qué se ha realizado en contextos similares. Para ello, puede ser conveniente llevar a cabo análisis de casos, indagando en los diversos repositorios de las organizaciones que se dedican a formular intervenciones conductuales.

Más allá de lo anterior, existe una serie de ideas o conceptos de las ciencias del comportamiento que se aplican de manera regular para determinadas barreras conductuales. Siguiendo este enfoque, el Behavioural Insights Team (BIT), la unidad de comportamiento del Reino Unido, ha desarrollado el marco EAST, una metodología para diseñar nudges, y que se basa en la idea de que las intervenciones deben ser Simples, Atractivas, Sociales y Oportunas (Service et al., 2014)

El principio de simplicidad sugiere, en primer lugar, aprovechar las opciones predeterminadas, valiéndose del sesgo del status-quo explicado anteriormente. De acuerdo a este principio, al convertir una opción en predeterminada, aumenta la probabilidad de que esta sea adoptada. De hecho, la evidencia indica que este tipo de nudges presentan los mayores impactos a la hora de modificar comportamientos (Hummel y Maedche, 2019). Algunos resultados conocidos de la aplicación de esta técnica son el incremento en el número de donantes de órganos (Abadie y Gay, 2006; Johnson y Goldstein, 2003) y de inscripciones en planes de pensiones (Madrian y Shea, 2001).

En segundo lugar, se propone reducir el esfuerzo (hassle factors) para acceder a un servicio, disminuyendo sus costos de transacción. Por ejemplo, simplificar el proceso de solicitud de beneficios sociales y acceso a la universidad para familias de bajos ingresos en los EE.UU ha demostrado tener impactos positivos en términos de incremento de las solicitudes de ayuda, inscripción en la universidad y acceso a más ayudas financieras (Bettinger et al., 2012). Del mismo modo, podrían pensarse intervenciones opuestas para desincentivar determinadas acciones.

En tercer lugar, se busca simplificar los mensajes destinados a los usuarios de un servicio, usando un lenguaje simple, siendo específico sobre las acciones a seguir, dando un solo punto de contacto o eliminando la información que no es estrictamente necesaria. La simplificación de los mensajes mejora las tasas de respuesta, reduce la carga de seguimiento, disminuye las consultas y los errores de comprensión. Por ejemplo, el rediseño de recetas médicas para hacerlas más simples ha reducido los errores en la prescripción de dosis (King et al., 2014).

El segundo principio requiere la intervención sea atractiva. En primer lugar, es necesario favorecer la prominencia, es decir, generar estímulos para que sobresalga determinada información. Algunas estrategias incluyen la personalización de mensajes, por ejemplo, incluyendo el nombre de las personas que mantienen deudas tributarias al solicitar la cancelación de las mismas o la fotografía del automóvil para alentar el pago de impuesto automotor (Service et al., 2014). No obstante, la personalización de mensajes puede insumir mayores costes a las Administraciones que apliquen estas intervenciones, por lo que deberá analizarse la relación coste-beneficio de las mismas (Castro y Scartascini, 2014).

En segundo lugar, dado que las personas son susceptibles a los incentivos y las sanciones, es posible reestructurarlos para potenciar sus efectos. En este sentido, vale aclarar que, si se modifican los incentivos, no se trataría de un nudge, ya que, precisamente, esa es una de las características indispensables.

\footnotetext{
6 La denominación EAST de este marco corresponde a la traducción inglesa de estos cuatro principios: Easy, Attractive, Social y Timely. Este marco de diseño es una evolución del enfoque MINDSPACE, también desarrollado por el BIT (Dolan et al., 2010).
} 
No obstante, si estos no se alteran sustancialmente o se estructuran de formas alternativas, pueden dar lugar a cambios en el comportamiento. El ejemplo más claro radica en la introducción de loterías. Si bien el premio de una lotería suele ser una suma considerable, capaz de inducir cambios en el comportamiento, el valor esperado del premio, es decir, la ganancia potencial ponderada por la probabilidad de ganar, es sumamente bajo, por lo que en realidad no debería modificar sustancialmente la estructura de incentivos (Carrillo et al., 2017).

Otros mecanismos para reestructurar incentivos comprenden la gamificación: utilizar juegos para involucrar a los usuarios en determinadas actividades y favorecer el alcance de objetivos mediante incentivos virtuales o tangibles, o aprovechar el deseo de las personas de mantener una imagen positiva de si mismas, ya que el incentivo de sentirse o verse bien, puede ser un elemento eficaz para motivar determinados comportamiento (Dolan et al., 2010).

El principio social es un elemento poderoso para orientar la conducta de las personas, por lo cual, los nudges buscan canalizarla a través de diferentes estrategias. Un primer mecanismo es describir el comportamiento de la mayoría de las personas, siempre que este sea positivo. Al conocer qué hacen las demás personas, se pone énfasis en el comportamiento esperado, lo que motiva a los demás a adoptarlo. Esta estrategia ha sido eficaz para mejorar la eficiencia en el consumo energético de los hogares (Allcott, 2011; Schultz et al., 2007).

También se recomienda utilizar las redes sociales para facilitar la acción colectiva, y proveer apoyo mutuo explotando el principio de reciprocidad entre las personas (Fehr y Gächter, 2000). Este mecanismo ha sido utilizado para incrementar las donaciones a partir de entregar pequeños regalos como un caramelo (Sanders, 2015). Asimismo, otra estrategia es promover los compromisos entre las personas. Al asumir compromisos con otros de manera voluntaria, la probabilidad de que la acción se lleve a cabo es mayor, dado que no hacerlo comporta un costo reputacional. Este mecanismo ha resultado eficaz, por ejemplo, para aumentar la actividad de búsqueda de trabajo por parte de solicitantes de empleo (Service et al., 2014).

Por último, los nudges deben ser oportunos, es decir, aplicarse en el momento en que las personas sean receptivas. Por ejemplo, el envío de recordatorios a través de SMS a los padres en determinados momentos del día, parece contrarrestar barreras conductuales que afectan la crianza de sus hijos (York y Loeb, 2018). Por otro lado, las personas son más propensas a cambiar sus hábitos durante periodos de transición, como por ejemplo luego de una mudanza, un casamiento, la pérdida de un familiar o al tener un hijo (Thompson et al., 2011). De este modo, es recomendable aprovechar estos momentos, en los cuales además se suele tomar contacto con la Administración, para aplicar intervenciones conductuales.

Por otro lado, deben considerarse los costos y beneficios de corto plazo. La miopía cognitiva, o sesgo del presente, hace que los costos y beneficios lejanos parezcan irrelevantes en el momento actual de toma de decisiones. Así, acciones que comprenden costos actuales o beneficios futuros son más difíciles de adoptar que otras que traen costos futuros o beneficios actuales. Por lo tanto, la idea es generar mecanismos para diferir los costos o traer al presente los beneficios lejanos de decisiones que resulten convenientes para las personas. De manera contraria, habría que traer al presente los costos o diferir los beneficios de acciones que puedan resultar perjudiciales. Como ejemplo de esto último, se han elaborado servicios para ayudar a los fumadores de dejar el hábito. Para ello, se les ofreció una cuenta de ahorros en la que depositar fondos durante seis meses. Pasado este plazo, se les realizaba una prueba para detectar nicotina y cotinina. Si superaban el test, se les devolvía el dinero (Giné et al., 2010).

También es recomendable ayudar a planificar respuestas ante determinadas situaciones. Debido a que existe una brecha entre las intenciones y el comportamiento real (Buehler et al., 1994), establecer un curso de acción anticipadamente a partir de un plan puede mejorar la toma de decisiones (Ariely y Wertenbroch, 2002). Por ejemplo, la venta de fertilizantes inmediatamente después de la cosecha, cuando los agricultores disponen de más recursos, con una entrega garantizada al momento en el momento necesario, ha incrementado su utilización aumentando la productividad agrícola en África Subsahariana (Duflo et al., 2011).

Una vez identificadas las posibles intervenciones o mecanismos que se utilizarán, deben diseñarse los tratamientos. En este sentido, es posible aplicar diversas versiones de una misma intervención, o diversas intervenciones, para analizar cuál es la más efectiva (Martínez Villarreal et al., 2020). Por ejemplo, si se ha llegado a la conclusión que la intervención más adecuada es simplificar los mensajes, pueden elaborarse diferentes alternativas que hagan énfasis en distintos aspectos para verificar cuál logra los mejores resultados. La selección de una u otra intervención dependerá de numerosos factores como el tiempo, los recursos disponibles o la potencial escalabilidad (Barrows et al., 2018). 
GAPP. Nueva Época - N. ${ }^{\circ}$ 25, marzo 2021 - ISSN: 1989-8991 - DOI: https://doi.org/10.24965/gapp.i25.10868 - [Págs. 8-22] Número monográfico - Los nudges y el diseño conductual de políticas públicas

Nudges: diseño y evaluación

Elena Costas Pérez / Pablo Tucat

Idealmente, las intervenciones deberían ser valoradas por un comité ético antes de aplicarse, porque conllevan riesgos éticos considerables y requieren en ocasiones de información personal sensible (OECD, 2019). Finalmente, en esta fase es sumamente relevante definir la estrategia de evaluación de impacto del programa.

\section{4. ¿POR QUÉ EVALUAR LOS NUDGES?}

El hecho de que los nudges sean acciones de bajo coste podría llevarnos a pensar que su evaluación no es necesaria. Sin embargo, como en cualquier política pública, tanto por eficiencia como por rendición de cuentas, es imprescindible poder medir su impacto. Una vez hemos definido el resultado deseado, el tipo de comportamiento necesario, y las potenciales intervenciones que nos permitirían conseguirlo, necesitamos verificar la eficiencia de los nudges. Es decir, cuantificar cómo consiguen modificar el comportamiento en la dirección deseada, e identificar posibles mejoras en su diseño y ejecución.

Por otro lado, es importante tener en cuenta que los nudges dependen en gran medida de los contextos en los que se apliquen, por lo que la prueba de estos mecanismos tiene que estar presente desde su propio diseño. Llevar a cabo pilotos de estas intervenciones puede ahorrar no sólo dinero, sino tiempo en la implementación en el contexto deseado. Nos permite también identificar efectos indeseados del propio nudge. Es por todas estas razones que los nudges son una de las intervenciones públicas que han implicado un mayor número de test y evaluaciones (Kosters y Van der Heijden, 2015).

Los nudges se basan en la ciencia del comportamiento para establecer su diseño y predicciones. Es un sólido punto de partida, pero no exento de imprecisiones. De hecho, en gran medida los nudges se basan en diseñar distintas opciones, y predecir las respuestas que se pueden dar ante cada una de ellas. Pero los propios «arquitectos» de este menú de opciones no estamos libres de estos sesgos. Por esa razón se hace imprescindible verificar de forma científica, en base a metodologías de evaluación, cuales son los resultados reales que provoca nuestra intervención (Behavioural Insights Team, 2010). Es decir, es necesario poder establecer la causalidad entre el nudge y el cambio de comportamiento observado.

Finalmente, más allá de identificar la eficiencia del nudge diseñado, su evaluación en una aplicación piloto nos permite también valorar hasta que punto merece la pena implementarlo a mayor escala. Es decir, se trata de medir el efecto del nudge, y valorar si éste es lo suficientemente grande.

\section{1. ¿Cómo evaluar los nudges?}

Muchas de las cuestiones mencionadas anteriormente se refieren al impacto de los nudges como política pública, y como tal, pueden evaluarse a través de una evaluación de este tipo. Como en cualquier otra política, el objetivo de la evaluación de impacto es el de identificar la causalidad entre la intervención y el resultado obtenido, y para ello contamos con diversas técnicas y metodologías rigurosas (Barrows et al., 2018).

Uno de los factores más relevantes al evaluar un nudge es su comparación con otras herramientas más tradicionales de implementar políticas, como podrían ser los incentivos fiscales o las multas. Como hemos visto, los nudges no pretenden forzar o prohibir comportamientos concretos, sino modificar el escenario sobre el que los ciudadanos toman decisiones. Esto supone un reto de cara a su evaluación, ya que el coste efectividad de los nudges puede no generar importantes cambios en el comportamiento ciudadano, lo que nos puede llevar a subestimar su impacto. Por lo tanto, es importante incluir también, al evaluar los nudges, un análisis coste-efectividad del impacto que genera cada euro invertido, en comparación con otras políticas más tradicionales que persiguen el mismo objetivo (Benartzi et al., 2017). Al tener en cuenta que se trata normalmente de intervenciones de muy bajo coste, aunque el impacto conseguido sea bajo, puede llegar a compensar el uso de políticas alternativas.

Por lo tanto, los nudges no tienen un sistema de evaluación específico, sino que se incluyen dentro de los métodos utilizados para cualquier política pública. Sin embargo, por su diseño y al estar en general basados en evidencia previa, los nudges permiten con más facilidad que otras intervenciones el uso de uno de los métodos de evaluación de impacto más riguroso -considerado como el gold standard- que son los ensayos controlados aleatorizados (RCT por sus siglas en inglés -randomized controlled trial-, también llamado A/B testing en algunos sectores) (Hopkins et al., 2020). De hecho, en el propio proceso de elaboración e implementación de nudges este tipo de evaluaciones constituye normalmente una fase esencial del (Haynes, Service, Goldacre y Torgerson, 2013; Service et al., 2014; Madaleno y Waights, 2016). 
Del mismo modo que se hace en los ensayos clínicos, la clave del enfoque experimental de los RCT en las ciencias sociales consiste en seleccionar a un colectivo de personas muy similares $\mathrm{y}$, de forma aleatoria, dar a unos participantes un tratamiento. En nuestro caso este tratamiento consistiría en la aplicación del nudge. El otro grupo sirve para controlar los efectos del tratamiento, por lo que los usuarios que lo componen no «son tratados». Para éstos observaríamos su comportamiento sin la intervención del nudge. De esta manera, la asignación al azar entre los dos grupos asegura que tanto el grupo de tratamiento como el grupo de control son equivalentes. Al ser estadísticamente idénticos, si tenemos grupos suficientemente grandes y considerando que hay un único cambio entre los sujetos de un grupo y del otro, el experimento no sólo permite medir el efecto de este estímulo -el nudge-, sino también establecer, hasta cierto punto, su causalidad. Cualquier cambio de comportamiento identificado se deberá únicamente al factor diferencial entre los dos grupos, es decir, el efecto del nudge (Glennerster y Takavarasha, 2013).

Las principales ventajas de esta metodología respecto a sus alternativas son el poder de control sobre la situación objeto de estudio y el correcto análisis de la causalidad. Se tiene también un grupo de comparación con el que cuantificar el efecto de la intervención. Con otros métodos de análisis es difícil establecer la causa-efecto del nudge debido, por ejemplo, a los distintos perfiles de los participantes (List et al., 2011). Por otro lado, los RCT son también fácilmente replicables (IRS, 2017).

Los RCTs no están sin embargo exentos de crítica. Es muy discutible la validez externa de los resultados obtenidos en estas evaluaciones, ya que los métodos experimentales dependen mucho del contexto en el que se aplican (IRS, 2017; Van Bavel et al., 2013). Lo que funciona en un territorio no tiene porqué funcionar en otro. También implican a veces cuestiones éticas que deben ser analizadas (Deaton, 2020).

Por otro lado, no siempre es posible llevar a cabo análisis experimentales. Puede ser que no tengamos una muestra suficientemente grande, lo que podría sesgar los resultados obtenidos, o que el RCT no se pueda llevar a cabo -por razones de coste, éticas o de implementación-. En esos casos, podemos contar con técnicas cuasi-experimentales, también conocidas como experimentos naturales (Homonoff et al., 2018). Se trata de aprovechar algún tipo de variación -geográfica, temporal o individual- con tal de verificar nuestra hipótesis de causalidad. En este caso no podremos contar con una aleatoriedad entre aquellos que están expuestos al nudge y los que no, por tanto, el reto se encuentra en identificar un buen grupo de comparación o control. En función del tipo de nudge y los datos que tengamos disponibles podemos recurrir a técnicas como diferencias en diferencias (DiD, por sus siglas en inglés), donde calculamos el efecto del tratamiento -el nudge-comparando los cambios de comportamiento en el grupo afectado por el nudge y el que no (Homonoff et al., 2018). Por ejemplo, si un fallo informático deja a varios usuarios sin el recordatorio de la declaración fiscal podemos evaluar el impacto de recibir información sobre el pago de impuestos (Manoli y Turner, 2014).

También es posible aprovechar algún tipo de discontinuidad en la asignación de una medida o política. Por ejemplo, analizar si el hecho de etiquetar una transferencia no condicionada, como el pago de combustibles -que se hace en función de la edad- hace aumenta el gasto en este tipo de bienes (Lange et al., 2018). Lo que queremos ver es si el hecho, supuestamente aleatorio, de caer a un lado u otro de la medida, para aquellas personas que se encuentran justo en el corte -y que, por tanto, deben ser muy similares- modifica de algún modo el comportamiento.

Existen otros métodos para evaluar los nudges de forma cuasi-experimental, como por ejemplo Propensity Score Matching (Castleman y Meyer, 2020), y elegir entre uno u otro depende principalmente de los datos a los que podamos tener acceso. A pesar de la mayor dificultad para establecer la relación casual entre el nudge y los cambios de comportamiento, estas metodologías nos pueden permitir una mayor representatividad del entorno que los métodos experimentales (List, 2007).

Por último, también tenemos métodos cualitativos, como los focus-group, las entrevistas estructuradas y semiestructuradas o la observación directa de los participantes. En este caso, no es posible establecer la causalidad entre el nudge y los posibles cambios de comportamiento observado, pero sí obtener una información valiosa sobre los mecanismos que pueden operar detrás de estos cambios (IRS, 2017; Van Bavel et al., 2013).

\section{2. ¿Qué sabemos sobre la utilidad de los nudges?}

Los nudges son medidas complementarias a otras políticas públicas, por lo que no nos centraremos aquí en determinar si este tipo de intervenciones son mejores que otras alternativas, como podría ser la regulación directa (Galle, 2014). Del mismo modo, la mayoría de estudios se centran en cuán efectivos son los nudges para modificar el comportamiento, sin tener en cuenta el coste -ya sea monetario o administrativoni su eficiencia (Kosters y Van der Heijden, 2015). 
Es importante tener en cuenta que, en su evaluación, no solo es relevante identificar cuál ha sido el impacto final obtenido, sino también qué tipo de cambios se pueden haber producido, por ejemplo en la forma en la que los actores interaccionan (Rogers y Weber, 2010) Estos datos son muy relevantes en la mejora del diseño de políticas públicas, incluso si se considera que el nudge no ha sido exitoso.

Tras varios lustros evaluando este tipo de intervenciones, los resultados de los nudges son diversos, pero en muchos casos se observa que, con intervenciones de muy bajo coste, los resultados obtenidos son muy beneficiosos, tanto para los propios individuos como el conjunto de la sociedad. Esto se suma al hecho principal de que nos permite analizar las políticas públicas desde una visión mucho más completa, al introducir otros factores, más allá de la racionalidad, que influyen en las decisiones que toman los ciudadanos.

Uno de los ejemplos más citados es el cambio en la opción de defecto de ser donante de órganos o no. Para los países europeos, en el caso que la donación sea la alternativa por defecto, el porcentaje de donantes es significativamente mayor que si esta opción se tiene que elegir expresamente. En palabras de los propios autores, las opciones predeterminadas salvan vidas (Johnson y Goldstein, 2003). De forma parecida, informar sobre el riesgo relativo de desarrollar una enfermedad individualmente, y no el del conjunto de la población, aumenta también nuestro interés al respecto (Lipkus et al., 2005).

Las evaluaciones constatan que el uso estratégico de la información puede modificar el comportamiento. Simplificar y acortar mensajes o personalizar recordatorios puede aumentar el pago de multas y facturas (Behavioural Insights Team, 2012). Algo similar ocurre con las normas sociales. Algo tan sencillo como añadir una carita triste al lado de nuestra factura de la luz si consumimos más que el resto, o comparando nuestro pago de impuestos con el del conjunto de la ciudad, parece reducir el consumo energético e incrementar la recaudación tributaria, respectivamente (Behavioural Insights Team, 2012; Schultz et al., 2007).

Al analizar alguna de las críticas realizadas a los nudges basadas en intervenciones que no han obtenido el resultado esperado (Kosters y Van der Heijden, 2015) vemos que, en muchos casos, al tratarse de un concepto tan amplio, se incluyen evaluaciones de experimentos que no necesariamente son nudges. Un claro ejemplo son las intervenciones que modifican los incentivos económicos, de los que ya se conoce que en muchos casos afectan a las motivaciones intrínsecas (Frey, 1994; Fryer Jr., 2011). Es decir, si pretendemos premiar a la gente por un comportamiento que harían igualmente de forma voluntaria, muchas veces se acaba desincentivando ese comportamiento al considerarse que ese pago es insuficiente (Kamenica, 2012). No obstante, al modificar los incentivos económicos, no estamos hablando de nudges precisamente.

Por otro lado, un resultado que se obtiene de la evaluación de los nudges es que en general no es recomendable combinar distintas estrategias - por ejemplo, la opción por defecto con campañas informativaspara conseguir un comportamiento concreto (Thaler y Sunstein, 2009). Tampoco podemos olvidar que los nudges pueden provocar comportamientos indeseados, como por ejemplo hacer que aquellos ciudadanos que se comportan mejor que la media empeoren sus hábitos una vez conocen esta información (Kosters y Van der Heijden, 2015; Schultz et al., 2007).

Uno de los trabajos más interesantes sobre el impacto de los nudges es un meta-análisis de 165 evaluaciones que afectan a más de 24 millones de personas (DellaVigna y Linos, 2020). El artículo analiza los proyectos llevados a cabo por dos de las principales unidades de economía del comportamiento y RCTs, el Behavioural Insights Team (BIT) y la Office of Evaluation Sciences (OES). Se trata de 345 intervenciones públicas de distintos niveles de gobierno, en áreas como salud, educación, o fiscalidad. Se consideraron todos los estudios de estas dos instituciones, tanto los publicados como los que no. De este análisis se obtiene un claro impacto positivo de los nudges. Los proyectos provocaron una mejora media del comportamiento superior al $8 \%$, a un bajo coste. De hecho, uno de los resultados más interesantes es que el menor coste de las intervenciones no supone en general un menor efecto del nudge. Ideas42 está realizando actualmente este tipo de meta-análisis con sus intervenciones, cuyos resultados nos permitirán un mayor conocimiento del efecto real de los nudges.

\section{REFLEXIONES FINALES}

En el diseño de políticas públicas hemos visto que los nudges constituyen un instrumento que permite tener en cuenta las limitaciones cognitivas de los ciudadanos al tomar decisiones, afectando así al entorno de su lógica decisoria con un bajo coste, pero cuya efectividad dependerá en gran medida de cómo se diseñen y cuáles sean los resultados de su evaluación. Ciertamente, los nudges no son una solución perfecta, la «bala de plata» (silver bullet) para resolver todo tipo de problemas sociales. En el primer ejemplo al que nos 
referíamos en este artículo, un nudge donde alimentos más saludables son destacados en el comedor de un colegio, puede hacer que los alumnos mejoren su dieta, pero no es una medida que aborda las dinámicas estructurales de la oferta de comida en nuestra sociedad, ni los factores socio-culturales responsables del reciente aumento de la obesidad en Occidente (Bhargava y Loewenstein, 2015).

Pero, por otro lado, los nudges pueden tener beneficios que van más allá de cambiar el comportamiento de los individuos, pues pueden ayudar a mejorar el funcionamiento de las propias administraciones. La adopción de una mentalidad abierta a la existencia de sesgos cognitivos puede ayudar a detectar errores sistemáticos en la adopción de muchas otras decisiones públicas, lo que abre un campo fértil tanto para gestores públicos como para investigadores (Battaglio et al., 2019). Así, por ejemplo, en decisiones políticas complejas a nivel ético o emocional, es importante complementar los conocimientos provenientes de la ciencia de datos con los hallazgos de la psicología humana de las ciencias del comportamiento como los nudges (Guszcza y Murphy, 2017). En una era marcada por el avance tecnológico, el big data y la Inteligencia Artificial (IA), la utilización de estas herramientas puede mejorar la toma de decisiones: a diferencia de los humanos, los algoritmos no experimentan racionalidad limitada, sesgos o fatiga cognitivos.

Los nudges han generado grandes expectativas. Muchos organismos públicos confían que «permitan a los decisores públicos entender mejor, e influir sobre, el comportamiento de la gente, [lo que] ayudará a diseñar políticas que funcionen mejor, cuesten menos y ayuden a la gente a conseguir sus metas» (European Commission, 2013). De momento, aunque los primeros indicios son buenos, carecemos de evidencia robusta que avale tales promesas (Kosters y Van der Heijden, 2015). De hecho, los nudges podrían sufrir la misma crisis de expectativas no cumplidas que el movimiento de la Nueva Gestión Pública a fines del siglo pasado (Pollit, 1995).

Sin embargo, y de forma paralela, tampoco parecen justificados los miedos contra el uso de los nudges. Según algunos autores, conllevan riesgos inaceptables dada la incertidumbre en torno a su efectividad. Pero descartarlos por ese miedo sería un error, porque los nudges se engarzan con una tendencia de largo recorrido que está mejorando la acción de gobierno en todo el mundo: la experimentación localizada. En este sentido, los nudges constituyen una importante herramienta de la política basada en la evidencia, mejorando así el diseño y efectividad de la acción pública. Como hemos intentado mostrar en este artículo, la revolución que traen los nudges no consiste tanto el acierto de su contenido concreto -los sesgos cognitivos que intentan corregir-, sino en su filosofía -basar el diseño de las políticas en el ensayo y el error-.

\section{REFERENCIAS BIBLIOGRÁFICAS}

Abadie, A. y Gay, S. (2006). The impact of presumed consent legislation on cadaveric organ donation: A cross-country study. Journal of Health Economics, 25(4), 599-620. https://doi.org/10.1016/j.jhealeco.2006.01.003

Abaluck, J. y Gruber, J. (2011). Choice inconsistencies among the elderly: Evidence from plan choice in the Medicare Part D program. American Economic Review, 101(4), 1.180-1.210. https://doi.org/10.1257/aer.101.4.1180

Afif, Z., Islan, W. W., Calvo-Gonzalez, O. y Dalton, A. G. (2018). Behavioral Science Around the World: Profiles of 10 Countries. World Bank Group. http://documents1.worldbank.org/curated/en/710771543609067500/pdf/132610REVISED-00-COUNTRY-PROFILES-dig.pdf

Allcott, H. (2011). Social norms and energy conservation. Journal of Public Economics, 95(9-10), 1.082-1.095. https:// doi.org/10.1016/j.jpubeco.2011.03.003

Ariely, D. y Loewenstein, G. (2006). The heat of the moment: The effect of sexual arousal on sexual decision making Journal of Behavioral Decision Making, 19(2), 87-98. https://doi.org/10.1002/bdm.501

Ariely, D. y Wertenbroch, K. (2002). Procrastination, deadlines, and performance: Self-control by precommitment. Psychological Science, 13(3), 219-224. https://doi.org/10.1111/1467-9280.00441

Barrows, A., Dabney, N., Hayes, J. y Rosenberg, R. (2018). Behavioral Design Teams. A Model for Integrating Behavioral Design in City Government. Ideas42. https://www.ideas42.org/wp-content/uploads/2018/04/BDT_ Playbook_FINAL-digital.pdf

Battaglio, R. P., Belardinelli, P., Bellé, N. y Cantarelli, P. (2019). Behavioral Public Administration ad fontes: A Synthesis of Research on Bounded Rationality, Cognitive Biases, and Nudging in Public Organizations. Public Administration Review, 79(3), 304-320. https://doi.org/10.1111/puar.12994

Behavioural Insights Team. (2010). Applying behavioural insight to health [Policy Paper]. The Cabinet Office. https:// www.gov.uk/government/publications/applying-behavioural-insight-to-health-behavioural-insights-team-paper

Behavioural Insights Team. (2012). Applying Behavioural Insights to Reduce Fraud, Error and Debt [Policy Paper]. The Cabinet Office. https://www.gov.uk/government/publications/fraud-error-and-debt-behavioural-insights-team-paper 
GAPP. Nueva Época - N. 25, marzo 2021 - ISSN: 1989-8991 - DOI: https://doi.org/10.24965/gapp.i25.10868 - [Págs. 8-22] Número monográfico - Los nudges y el diseño conductual de políticas públicas

Nudges: diseño y evaluación

Elena Costas Pérez / Pablo Tucat

Benartzi, S., Beshears, J., Milkman, K. L., Sunstein, C. R., Thaler, R. H., Shankar, M., Tucker-Ray, W., Congdon, W. J. y Galing, S. (2017). Should Governments Invest More in Nudging? Psychological Science, 28(8), 1.041-1.055. https://doi.org/10.1177/0956797617702501

Benartzi, S. y Thaler, R. H. (2007). Heuristics and biases in retirement savings behavior. Journal of Economic Perspectives, 21(3), 81-104. https://doi.org/10.1257/jep.21.3.81

Bettinger, E. P., Long, B. T., Oreopoulos, P. y Sanbonmatsu, L. (2012). The Role of Application Assistance and Information in College Decisions: Results from the H\&R Block FAFSA Experiment. Quarterly Journal of Economics, 127(3), 1.205-1.242. https://doi.org/10.1093/qje/qjs017

Bhargava, S. y Loewenstein, G. (2015). Behavioral economics and public policy 102: Beyond nudging. American Economic Review, 105(5), 396-401. https://doi.org/10.1257/aer.p20151049

Buehler, R., Griffin, D. y Ross, M. (1994). Exploring the «Planning Fallacy»: Why People Underestimate Their Task Completion Times. Journal of Personality and Social Psychology, 67(3), 366-381. https://web.mit.edu/curhan/www/ docs/Articles/biases/67_J_Personality_and_Social_Psychology_366,_1994.pdf

Carrillo, P. E., Castro, E. y Scartascini, C. (2017). Do Rewards Work?: Evidence from the Randomization of Public Works (IDB Working Paper 794). Inter-American Development Bank. https://publications.iadb.org/en/do-rewardswork-evidence-randomization-public-works

Castleman, B. L. y Meyer, K. E. (2020). Can Text Message Nudges Improve Academic Outcomes in College? Evidence from a West Virginia Initiative. The Review of Higher Education, 43(4), 1.125-1.165. https://doi.org/10.1353/ rhe.2020.0015

Castro, L. y Scartascini, C. (2014). El diablo está en los detalles. Algunas lecciones para el diseño de políticas públicas. Resumen de Políticas, IDB-PB-232. Banco Interamericano de Desarrollo. https://publications.iadb.org/es/ publicacion/16875/el-diablo-esta-en-los-detalles-algunas-lecciones-para-el-diseno-de-politicas

Davies, H. T. O., Nutley, S. M. y Smith, P. C. (2000). What works? Evidence-based policy and practice in public services. Bristol University Press. https://doi.org/10.2307/j.ctt1t892t3

De Gilder, D., Schuyt, T. N. M. y Breedijk, M. (2005). Effects of an Employee Volunteering Program on the Work Force: The ABN-AMRO Case. Journal of Business Ethics, 61(2), 143-152. Springer. https://doi.org/10.1007/s10551-0057101-x

Deaton, A. (2020). Randomization in the Tropics Revisited: a Theme and Eleven Variations (NBER Working Paper, 27.600). National Bureau of Economic Research. https://doi.org/10.3386/w27600

DellaVigna, S. y Linos, E. (2020). RCTs to Scale: Comprehensive Evidence from Two Nudge Units (NBER Working Paper, 27.594). National Bureau of Economic Research. https://doi.org/10.3386/w27594

Dhami, S. (2016). The foundations of behavioral economic analysis. Oxford University Press.

Dolan, P., Hallsworth, M., Halpern, D., King, D. y Vlaev, I. (2010). MINDSPACE: Influencing behaviour through public policy. Institute of Government. https://www.instituteforgovernment.org.uk/sites/default/files/publications/ MINDSPACE.pdf

Duflo, E., Kremer, M. y Robinson, J. (2011). Nudging farmers to use fertilizer: Theory and experimental evidence from Kenya. American Economic Review, 101(6), 2.350-2.390. https://doi.org/10.1257/aer.101.6.2350

Einfeld, C. (2019). Nudge and evidence based policy: fertile ground. Evidence \& Policy: A Journal of Research, Debate and Practice, 15(4), 509-524. https://doi.org/10.1332/174426418X15314036559759

European Commission (2013). Vulnerable Consumer Working Group Guidance Document on Vulnerable Consumers, November 2013. Vulnerable Consumer Working Group. https://ec.europa.eu/energy/sites/ener/files/ documents/20140106_vulnerable_consumer_report_0.pdf

Fehr, E. y Gächter, S. (2000). Fairness and Retaliation: The Economics of Reciprocity. The Journal of Economic Perspectives, 14(3), 159-181. American Economic Association. https://doi.org/10.2307/2646924

Frey, B. S. (1994). How Intrinsic Motivation is Crowded out and in. Rationality and Society, 6(3), 334-352. https://doi. org/10.1177/1043463194006003004

Fryer Jr., R. G. (2011). Teacher Incentives and Student Achievement: Evidence from New York City Public Schools (NBER Working Paper, 16.850). National Bureau of Economic Research. https://doi.org/10.3386/w16850

Galle, B. (2014). Tax, Command... or Nudge? Evaluating the New Regulation. Texas Law Review, 92(4), 837-894. http://texaslawreview.org/wp-content/uploads/2015/08/Galle-92-4.pdf

García Arteagoitia, S. (2019). El éxito y el potencial del nudge en las políticas públicas. Revista Vasca de Gestión de Personas y Organizaciones Públicas, (3 Especial), 140-155. https://www.ivap.euskadi.eus/contenidos/informacion/ rvgp_ultimo_numero/es_def/Garc\%C3\%ADa\%20140_155.pdf

Giné, X., Karlan, D. y Zinman, J. (2010). Put your money where your butt is: A commitment contract for smoking cessation. American Economic Journal: Applied Economics, 2(4), 213-235. https://doi.org/10.1257/app.2.4.213

Glennerster, R. y Takavarasha, K. (2013). Running randomized evaluations: A practical guide. Princeton University Press.

Guszcza, J. y Murphy, T. (2017). Cognitive Collaboration: What Data Science Can Learn from Psychology. En A. Samson (Ed.), The Behavioral Economics Guide 2017 (pp. 41-46).

Haynes, L., Service, O., Goldacre, B. y Torgerson, D. (2013). Test, Learn, Adapt: Developing Public Policy with Randomised Controlled Trials. The Cabinet Office. Behavioural Insights Team. https://www.bi.team/publications/ test-learn-adapt-developing-public-policy-with-randomised-controlled-trials/ 
GAPP. Nueva Época - N. 25, marzo 2021 - ISSN: 1989-8991 - DOI: https://doi.org/10.24965/gapp.i25.10868 - [Págs. 8-22] Número monográfico - Los nudges y el diseño conductual de políticas públicas

Nudges: diseño y evaluación

Elena Costas Pérez / Pablo Tucat

Hershfield, H. E., Goldstein, D. G., Sharpe, W. F., Fox, J., Yeykelis, L., Carstensen, L. L. y Bailenson, J. N. (2011). Increasing saving behavior through age-progressed renderings of the future self. Journal of Marketing Research, 48(SPL), S23-S37. https://doi.org/10.1509/jmkr.48.SPL.S23

Homonoff, T., Kao, L.-S., Palmer, D. y Seybolt, C. (2018). Skipping the bag. Assessing the impact of Chicago's tax on disposable bags. NYU Wagner. https://wagner.nyu.edu/impact/research/publications/skipping-bag-assessingimpact-chicagos-tax-disposable-bags

Hopkins, A., Breckon, J. y Lawrence, J. (2020). The Experimenter's Inventory. A catalogue of experiments for decisionmakers and professionals. Alliance for Useful Evidence is a network - Nesta. https://www.alliance4usefulevidence. org/assets/2020/01/The-Experimenters-Inventory-A-catalogue-of-experiments-for-decision-makers-andprofessionals.pdf.pdf

Hummel, D. y Maedche, A. (2019). How effective is nudging? A quantitative review on the effect sizes and limits of empirical nudging studies. Journal of Behavioral and Experimental Economics, 80, 47-58. https://doi.org/10.1016/j. socec.2019.03.005

Internal Revenue Service (2017). Behavioral Insights Toolkit. IRS. https://www.irs.gov/pub/irssoi/17rpirsbehavioralinsights.pdf

lyengar, S. S. y Lepper, M. R. (2000). When choice is demotivating: can one desire too much of a good thing? Journal of Personality and Social Psychology, 79(6), 995-1.006. https://doi.org/10.1037/0022-3514.79.6.995

Johnson, E. J. y Goldstein, D. (2003). Do Defaults Save Lives? Science, 302(5.649), 1.338-1.339. https://doi. org/10.1126/science.1091721

Kahneman, D. (1994). New Challenges to the Rationality Assumption. Journal of Institutional and Theoretical Economics, 150(1), 18-36. https://www.jstor.org/stable/40753012

Kahneman, D. (2011). Thinking, Fast and Slow (edición 2013). Macmillan.

Kahneman, D. y Tversky, A. (1972). Subjective probability: A judgment of representativeness. Cognitive Psychology, 3(3), 430-454. https://doi.org/10.1016/0010-0285(72)90016-3

Kahneman, D. y Tversky, A. (1979). Prospect theory: An analysis of decision under risk. Econometrica, 47(2), $263-292$. https://doi.org/10.2307/1914185

Kamenica, E. (2012). Behavioral Economics and Psychology of Incentives. Annual Review of Economics, 4(1), 427452. https://doi.org/10.1146/annurev-economics-080511-110909

King, D., Jabbar, A., Charani, E., Bicknell, C., Wu, Z., Miller, G., Gilchrist, M., Vlaev, I., Franklin, B. D. y Darzi, A. (2014). Redesigning the «choice architecture» of hospital prescription charts: A mixed methods study incorporating in situ simulation testing. BMJ Open, 4(12), 5.473. https://doi.org/10.1136/bmjopen-2014-005473

Kosters, M. y Van der Heijden, J. (2015). From mechanism to virtue: Evaluating Nudge theory. Evaluation, 21(3), 276291. https://doi.org/10.1177/1356389015590218

Lange, I., Moro, M. y Mahbubur Rahman, M. (2018). Cleaner Nudges? Policy Labels and Investment Decision-making. The Energy Journal, 39(6). https://doi.org/10.5547/01956574.39.6.ilan

Lindblom, C. E. (1959). The Science of «Muddling Through». Public Administration Review, 19(2), 79-88. https://doi. org/10.2307/973677

Lipkus, I., Klein, W., Skinner, C. S. y Rimer, B. (2005). Breast cancer risk perceptions and breast cancer worry: What predicts what? Journal of Risk Research, 8(5), 439-452. https://doi.org/10.1080/1366987042000311018

List, J. A. (2006). Field Experiments: A Bridge between Lab and Naturally Occurring Data. The B.E. Journal of Economic Analysis \& Policy, 5(2). https://doi.org/10.2202/1538-0637.1747

List, J. A., Sadoff, S. y Wagner, M. (2011). So you want to run an experiment, now what? Some simple rules of thumb for optimal experimental design. Experimental Economics, 14(4), 439-457. https://doi.org/10.1007/s10683-0119275-7

Madaleno, M. y Waights, S. (2016). Guide to scoring methods using the Maryland Scientific Methods Scale. What Works Centre for Local Economic Growth. https://whatworksgrowth.org/public/files/Scoring-Guide.pdf

Madrian, B. C. y Shea, D. F. (2001). The power of suggestion: Inertia in 401 (k) participation and savings behavior. The Quarterly Journal of Economics, 116(4), 1.149-1.187. https://www.jstor.org/stable/2696456

Manoli, D. y Turner, N. (2014). Nudges and Learning: Evidence from Informational Interventions for Low-Income Taxpayers (NBER Working Paper, 20.718). National Bureau Of Economic Research. https://doi.org/10.3386/ w20718

Martínez Villarreal, D., Rojas Méndez, A. M. y Scartascini, C. (2020). La economía del comportamiento puede ayudar a combatir el coronavirus. Resumen de Políticas, IDB-PB-334. Banco Interamericano de Desarrollo. http://dx.doi. org/10.18235/0002315

Mullainathan, S. y Shafir, E. (2013). Scarcity: Why having too little means so much. Times Books. http://sc.arcity.co/

Nisbett, R. E. y Wilson, T. D. (1977). The Halo Effect: Evidence for Unconscious Alteration of Judgments. Journal of Personality and Social Psychology, 35(4), 250-256. https://doi.org/10.1037/0022-3514.35.4.250

O’Donoghue, T. y Rabin, M. (1999). Doing It Now or Later. American Economic Review, 89(1), 103-124. https://doi. org/10.1257/aer.89.1.103

OECD (2019). Tools and Ethics for Applied Behavioural Insights: The BASIC Toolkit. OECD Publishing. https://doi. org/10.1787/9ea76a8f-en 
Pallier, G., Wilkinson, R., Danthiir, V., Kleitman, S., Knezevic, G., Stankov, L. y Roberts, R. D. (2002). The role of individual differences in the accuracy of confidence judgments. Journal of General Psychology, 129(3), $257-299$. https://doi.org/10.1080/00221300209602099

Pollitt, C. (1995). Justification By Works Or By Faith? Evaluating The New Public Management. Evaluation, 1(2), 133154. https://doi.org/10.1177/135638909500100202

Rogers, E. y Weber, E. P. (2010). Thinking Harder About Outcomes for Collaborative Governance Arrangements. The American Review of Public Administration, 40(5), 546-567. https://doi.org/10.1177/0275074009359024

Sabel, C. F. y Zeitlin, J. (2012). Experimentalist Governance. The Oxford Handbook of Governance, 1, 2-4. https://doi. org/10.1093/oxfordhb/9780199560530.013.0012

Samson, A. (ed.) (2020). The Behavioral Economics Guide 2020 (con introducción de Colin Camerer). Behavioral Science Solutions. https://www.behavioraleconomics.com/be-guide/the-behavioral-economics-guide-2020/

Samuelson, W. y Zeckhauser, R. (1988). Status quo bias in decision making. Journal of Risk and Uncertainty, 1(1), 7-59. https://doi.org/10.1007/BF00055564

Sanders, M. (2015). In search of the limits of applying reciprocity in the field: Evidence from two large field experiments. The Behavioural Insights Team and Harvard Kennedy School of Government. https://www.bi.team/publications/insearch-of-the-limits-of-applying-reciprocity-in-the-field-evidence-from-two-large-field-experiments/

Schultz, P. W., Nolan, J. M., Cialdini, R. B., Goldstein, N. J. y Griskevicius, V. (2007). The Constructive, Destructive, and Reconstructive Power of Social Norms. Psychological Science, 18(5), 429-434. https://www.jsmf.org/ meetings/2008/july/social\%20norms\%20Cialdini.pdf

Service, O., Hallsworth, M., Halpern, D., Algate, F., Gallagher, R., Nguyen, S., Ruda, S., Sanders, M. with Marcos Pelenur, M., Gyani, A., Harper, H., Reinhard, J. y Kirkman, E. (2014). EAST: Four simple ways to apply behavioural insights. The Behavioural Insights Team. https://www.bi.team/publications/east-four-simple-ways-to-applybehavioural-insights/

Shafir, E. (2007). A behavioural background for economic policy. Behavioural Economics and Public Policy. Australian Government Productivity Commission. https://www.pc.gov.au/research/supporting/behavioural-economics/shafir. pdf

Sharot, T. (2011). The optimism bias. Current Biology, 21(23), R941-R945. https://doi.org/10.1016/j.cub.2011.10.030

Simon, H. A. (1956). Rational choice and the structure of the environment. Psychological Review, 63(2), 129-138. https://doi.org/10.1037/h0042769

Straßheim, H. y Beck, S. (eds.). (2019). Handbook of Behavioural Change and Public Policy. (Handbooks of Research on Public Policy series) Edward Elgar Publishing. https://doi.org/10.4337/9781785367854

Straßheim, H., Jung, A. y Korinek, R.-L. (2015). Reframing expertise: The rise of behavioural insights and interventions in public policy. En A. Berthoin Antal, M. Hutter y D. Stark (eds.), Moments of valuation. Exploring sites of dissonance (pp. 249-268). Oxford University Press. https://doi.org/10.1093/acprof:oso/9780198702504.003.0013

Thaler, R. H. y Sunstein, C. R. (2009). Nudge: Improving Decisions About Health, Wealth, and Happiness. Yale University Press.

Thompson, S., Michaelson, J., Abdallah, S., Johnson, V., Morris, D., Riley, K. y Simms, A. (2011). «Moments of change» as opportunities for influencing behaviour: A report to the Department for Environment, Food and Rural Affairs, nef (the new economics foundation). Defra. https://core.ac.uk/reader/9717890

Tversky, A. y Kahneman, D. (1974). Judgment under uncertainty: Heuristics and biases. Science, 185(4.157), 1.1241.131. https://doi.org/10.1126/science.185.4157.1124

Van Bavel, R., Herrmann, B., Esposito, G. y Proestakis, A. (2013). Applying Behavioural Sciences to EU Policymaking. JRC Scientific and Policy Reports (JRC83284). Publications Office of the European Union. https://doi. org/10.2788/47172

York, B. y Loeb, S. (2018). One Step at a Time: The Effects of an Early Literacy Text Messaging Program for Parents of Preschoolers (NBER Working Paper, 20.659). National Bureau of Economic Research. https://doi.org/10.3386/ w20659 